high and low density lipoprotein (HDL and LDL) and liver function profiles (Alanine aminotransferase (ALT), alkaline phosphatase (ALP) and Aspartate aminotransferase (AST)). Hepatoprotective activity, Hepatosomic Index (HI) and histopathological changes in liver tissues were also evaluated.

Results Animals administered with honey showed significant $(\mathrm{p}<0.05)$ decrease on TC, TG, LDL and significant increase $(p<0.05)$ of HDL when compared to HFD. Interestingly, honey supplementation exhibited a hepatoprotective effects as indicated by reduced ALT and AST levels and HI index. Adipocytokines were markedly ameliorated in HFDPH compared to HFD. Adiponectin levels significantly increased in HFDAH and HFDPH (8.13 and $8.22 \mathrm{ug} / \mathrm{mL}$ respectively) compared to HFDO $(6.07 \mathrm{ug} / \mathrm{mL})$. Leptin level $(\mathrm{p}<0.05)$ decreased from 8.08 to $5.68 \mathrm{ng} / \mathrm{mL}$ for the HFDPH compared to HFD. Histopathological changes were observed in the liver tissue of HFD and HFDO treated group. The honey supplementation restored all the biochemical parameters significantly $(\mathrm{p}<0.05)$, especially the liver function parameters and histopathological changes when compared to HFD or orlistat.

Conclusions In conclusion, honey supplementation has positive effects on modulating several biochemical pathways of adipocytokine excretions, lipid profiles as well as hepatoprotective which may potentially be applicable for suppression of obesity and its metabolic complications.

\section{Clinical Gastroenterology}

\section{IDDF2018-ABS-0026 PERSISTENCE AND ADHERENCE OF ANTI- TUMOUR NECROSIS FACTOR (TNF) BIOLOGICS FOR INFLAMMATORY BOWEL DISEASE (IBD) IN AUSTRALIA}

${ }^{1}$ Li-Chin Wang*, ${ }^{2}$ Rupert Leong. ${ }^{1}$ Faculty of Medicine, University of Sydney, Australia; ${ }^{2}$ Concord Repatriation Hospital, Gastroenterology and Liver Services, Australia

10.1136/gutjnl-2018-IDDFbestabstracts. 10

Background Infliximab (IFX) and adalimumab (ADA) induce and sustain IBD clinical remission, but their adherence and persistence are yet to be characterised in the Australian population. These markers are surrogates for treatment efficacy and determinants of cost-effectiveness. We analysed real-world dispensing of IFX and ADA for IBD in Australia using national Pharmaceutical Benefits Scheme (PBS) data.

Methods 10 year national PBS data obtained (2005-2015). Non-persistence defined as no ADA and IFX prescriptions for 6 and 7 months respectively. Kaplan-Meier analysis conducted with Cox regression adjusting for age, gender and state. Adherence defined as mean possession ratio (MPR) >0.8, with yearly MPR and Chi-squared analysis. Initiation and maintenance phases determined by PBS codes with dose-intervals corrected.

Results Persistence for IFX and ADA: $77 \%$ and $79 \%$ (6 months), 68\% and 69\% (12 months), and 50\% and 52\% (3 years) respectively (IDDF2018-ABS-0026 Figure 1. Kaplan meier curve of persistence for ifx-and ada); no statistical differences in persistence observed (hazard ratio [HR]: 1.03, 95\% CI: $0.75-1.43, p=0.84$, table 1$)$. Those younger than 20 (HR: 2.08, 95\% CI: 1.15-3.74, $\mathrm{p}=0.02$ ) and older than 61 (HR: $1.99,95 \% \mathrm{CI}: 1.11-3.59, \mathrm{p}=0.02$ ) had double discontinuation risks than reference age (41-50). Patients in Victoria had greater persistence than New South Wales (HR: 0.65,
95\% CI: 0.43-0.99, $\mathrm{p}=0.04)$. Maximal discontinuation within first 1-6 months (IFX 23\%, ADA 17\%). IFX had higher MPR than ADA (IDDF2018-ABS-0026 Figure 2. Mean yearly mpr for ifx and ada): year 1: 0.92, $0.79(\mathrm{p}<0.001)$; year 2: $0.93,0.86(p=0.012)$, year $3: 0.99,0.94 \quad(p=0.047) .65 \%$ and $84 \%$ of patients were adherent for ADA and IFX respectively $(p<0.001)$; differences driven by initiation therapy.

\begin{tabular}{lll}
$\begin{array}{l}\text { Abstract IDDF2018-ABS-0026 Table 1 } \\
\text { for Persistence P 0-0 }\end{array}$ & Cox Regression Analysis \\
\hline & Univariate analysis & Multivariate analysis \\
\hline IFX (ref= ADA) & HR $1.03(0.75-1.43)$ & HR $1.03(0.74-1.44)$ \\
Male (ref= F) & HR $1.24(0.90-1.72)$ & HR $1.18(0.85-1.64)$ \\
Age (ref $41-50)$ & HR $2.08(1.15-3.74) *$ & \\
$0-20$ & HR $1.41(0.86-2.32)$ & $<30:$ HR $1.47(0.92-2.36)$ \\
$21-30$ & HR $1.31(0.77-2.23)$ & HR $1.28(0.75-2.19)$ \\
$31-40$ & 1 & 1 \\
$41-50$ & HR $1.53(0.79-2.94)$ & HR $1.47(0.76-2.85)$ \\
$51-60$ & HR $1.99(1.11-3.59) *$ & HR $1.95(1.08-3.52)$ \\
$61+$ & &
\end{tabular}

Conclusions Persistence and adherence for anti-TNFs among Australian IBD sufferers are suboptimal, with better adherence for IFX than ADA. There was comparable persistence between the biologics, with poorer persistence in the elderly suggesting its use as bridging therapy for surgery to avoid long-term immunosuppression. Discontinuation was determined by the first six months, with geographical heterogeneity identified. Personalised interventions should be developed to improve adherence through anti-TNF selection, education, empowerment and follow-up.

\section{IDDF2018-ABS-0041 STRUCTURAL CHANGES IN THE HUMAN GUT MICROBIOME FOLLOWING TRIPLE THERAPY USING A POTASSIUM- COMPETITIVE ACID BLOCKER IN HELICOBACTER PYLORI-INFECTED JUNIOR HIGH-SCHOOL STUDENTS}

${ }^{1}$ Takuji Gotoda* ${ }^{2}$ Chika Takano, ${ }^{1}$ Chika Kusano, ${ }^{1}$ Sho Suzuki, ${ }^{1}$ Hisatomo Hehara, ${ }^{3}$ Satoshi Hayakawa, ${ }^{4}$ Akira Andoh. 'Division of Gastroenterology and Hepatology, Department of Medicine, Nihon University School of Medicine, Japan; ${ }^{2}$ Department of Pediatrics and Child Health, Nihon University School of Medicine, Japan; ${ }^{3}$ Division of Microbiology, Department of Pathology and Microbiology, Nihon University School of Medicine, Japan; ${ }^{4}$ Division of Gastroenterology, Department of Medicine, Shiga University of Medical Science, Japan

\subsection{6/gutjnl-2018-IDDFbestabstracts. 11}

Background Helicobacter pylori eradication is imperative to prevent gastric cancer before the development of gastric atrophy and/or intestinal metaplasia. However, there is no clear consensus on the optimal age for $H$. pylori eradication, and changes in the gut microbiota after eradication and their potential future impacts remain unknown. Our aim was to assess gut microbiome changes after $H$. pylori eradication in children.

Methods Changes in the gut microbiota before and after $H$. pylori eradication was prospectively investigated in 8 students without any underlying diseases by $16 \mathrm{~s}$ rRNA genes using next-generation sequencing. A total of 24 stool samples were collected, and operational taxonomic unit analysis was performed. As secondary analyses, alpha and beta diversity were 